\title{
Research Progress of Cognitive Impairment in Post-Traumatic Stress Disorder
}

\author{
Liumei Luo, Yanhai Kang, Fanchang Zeng, Yuzhu Lin, Ying Feng, Mengmeng Li, Feiyan Li* \\ Hainan General Hospital, Haikou, China \\ Email: ^g2002m@163.com
}

How to cite this paper: Luo, L.M., Kang, Y.H., Zeng, F.C., Lin, Y.Z., Feng, Y., Li, M.M. and Li, F.Y. (2021) Research Progress of Cognitive Impairment in Post-Traumatic Stress Disorder. Health, 13, 157-164. https://doi.org/10.4236/health.2021.132014

Received: January 14, 2021

Accepted: February 20, 2021

Published: February 23, 2021

Copyright $\odot 2021$ by author(s) and Scientific Research Publishing Inc. This work is licensed under the Creative Commons Attribution International License (CC BY 4.0).

http://creativecommons.org/licenses/by/4.0/

\begin{abstract}
Post-traumatic stress disorder (PTSD) is a kind of serious mental disorder that occurs after severe traumatic events. It is characterized by severe emotional and memory damage. This paper reviews the relevant research literature on PTSD at home and abroad in recent years, and reviews the mechanism of cognitive impairment in mental trauma to reveal the functional mechanism of cognitive impairment in post-traumatic stress disorder, in order to provide reference for future research.
\end{abstract}

\section{Keywords}

PTSD, Cognitive Function, Mechanism, Review

\section{Introduction}

In recent years, with the frequent occurrence of natural and man-made disasters, a series of malignant events have not only caused great loss of life and property, but also posed a major threat to people's physical and mental health. Among them, post-traumatic stress disorder is one of the serious consequences of these injuries. Post-Traumatic Stress Disorder (PTSD) is a disorder in which a person who has directly experienced a traumatic event develops a characteristic set of symptoms (intrusion/re-experiencing the trauma; avoidance; negative cognitions and mood; hyper-arousal) [1]. A survey on the incidence of dementia in a large number of veterans in the United States showed that the risk of dementia in veterans with PTSD was about twice than in veterans without PTSD [2] [3] [4]. It is not clear whether there are common risk factors for PTSD and dementia, or whether PTSD itself is an independent risk factor for dementia. However, the current research on PTSD is mainly based on clinical observation, while the research on the mechanism of PTSD is still not in-depth enough. The author 
summarizes the mechanism of brain function of PTSD by combining and summarizing the research literature on the mechanism of PTSD in recent years, in order to provide reference for future related research.

\section{Abnormal Changes in Mood}

Brain is not only the highest organ regulating stress, but also an important target organ of stress. At present, it is generally believed that the amygdala plays an important role in emotion and emotion regulation, and the enhancement of its activity may cause excessive fear response or impaired fear memory in patients [5]. Brunetti et al. [6] used fMRI technology to find that the activation process of the amygdala in adult PTSD patients was abnormally increased under neutral or negative stimuli. However, Liu Mengqi et al. [7] firstly studied the neurobiochemical changes of bilateral amygdala in children and adolescents with PTSD by using MRS technique, and preliminarily discussed its possible role in the pathogenesis of PTSD. The study found that the left amygdala creatine (CR) concentration was significantly higher than that of the right amygdala in the healthy control group, suggesting that the distribution of CR concentration in the normal children and adolescents was asymmetrical. There was no significant difference in the concentration of $\mathrm{Cr}$ in the left amygdala of the PTSD group compared with the healthy control group. $\mathrm{Cr}$ is a buffer of high-energy phosphate in energy metabolism, and previous studies have shown that the concentration of $\mathrm{Cr}$ will decrease in the high metabolic state, and, on the contrary, it will increase in the low metabolic state [8]. This study suggested that the asymmetry of $\mathrm{Cr}$ concentration distribution in the amygdala of PTSD patients was destroyed, and the left amygdala was hypermetabolic. Therefore, it is speculated that the activation of the amygdala (especially the left amygdala) is abnormally increased under the stimulation of traumatic events, resulting in high metabolism, thus breaking the asymmetry of the distribution of $\mathrm{Cr}$ concentration in the amygdala, which may be one of the mechanisms of the recurrence of traumatic experience and the increase of persistent alertness in PTSD patients. Karl et al. [9] found in their Meta-analysis that the bilateral amygdala in PTSD patients was significantly reduced, and the left amygdala volume was significantly reduced in PTSD patients compared with normal healthy people, which proved on the other hand that the abnormal changes in the structure and function of the amygdala (especially the left amygdala) were closely related to the occurrence of PTSD. Rauch et al. [10] found that the amygdala cerebral blood flow in patients with PTSD increased in the fearful imagination environment, and decreased in the amygdala cerebral blood flow after a period of CBT. It is suggested that the increased amygdala activity is an important pathological basis of PTSD. At the same time, Rauch et al. [10] also found that the blood flow in the bilateral insula of PTSD patients increased, and the insula has extensive fibrous connections with many brain regions, such as the frontal lobe, temporal lobe, amygdala, and hippocampus, which is an important node in the emotional processing and au- 
tonomic regulation network. Therefore, it is speculated that the activation of the insula may also be closely related to the occurrence of PTSD. Many domestic and foreign studies [11] [12] [13] [14] [15] believe that the consistency of brain precuneus in patients with PTSD is abnormally activated, suggesting that the local neuronal activity of precuneus in patients with resting state is enhanced in synchronization, indicating hyperfunction. Studies have shown that the precuneus is an important part for processing visual information [16] and is involved in extracting emotional memories [17]. It is speculated that the enhancement of precuneus function may be one of the important mechanisms of recurrent traumatic experience in PTSD patients.

\section{Abnormal Changes in Memory Function}

PTSD is often associated with memory impairment. Studies have shown that both the frontal and temporal lobes of the brain are directly involved in the regulation of memory. The frontal lobe is responsible for the integration of all senses and perception, the frontal cortex is involved in memory formation, and the temporal cortex is involved in memory formation and retrieval [18]. Gong Li et al. [19] used MRI technology to investigate the correlation between PTSD memory impairment and brain structure, and found that the scores of understanding of memory, delayed understanding memory, visual regeneration and delayed visual regeneration in PTSD patients were significantly lower than those in normal healthy people, suggesting that PTSD patients had different degrees of memory impairment. MRI results showed that the volume of gray matter in the frontal and temporal lobes of the patients with PTSD was significantly smaller than that of normal healthy people. It is preliminarily suggested that the memory impairment of PTSD is closely related to the structural changes of the frontal and temporal lobes. When Jin Kuixing et al. [20] studied the correlation between executive function of early PTSD and frontal lobe damage, they found that PTSD patients had obvious executive dysfunction and the gray matter volume of part of the frontal lobe decreased, which was similar to the conclusion that frontal lobe damage might be the pathological basis of cognitive function damage. The reason may be that the frontal lobe damage weakens the inhibitory effect on the amygdala, thus enhancing its own activity, affecting the normal memory processing and ultimately affecting the encoding, recognition and recognition of declarative memory. Director of learning and memory function in temporal lobe area as the hippocampus, also have scholars launched a related study, Min Guo, etc. [21]. The MRI hippocampal volume of 50 patients with acute PTSD was compared with that of 50 healthy volunteers, Normal hippocampal volume was correlated with age and sex. This study used the difference method to measure the hippocampal volume of correction, and matched controls in terms of age, gender, as far as possible to avoid the error caused by objective factors. Then they found that people with PTSD hippocampal volume compared with healthy controls were changed, but to different degree, patients with mild-to-moderate 
hippocampal volume shrink are not obvious. The difference was not statistically significant, but the volume reduction in severe patients was significantly differ-

ent [22]. It is suggested that the occurrence of PTSD is related to the damage of hippocampus, and it is speculated that the degree of hippocampus volume reduction may be related to the severity of PTSD patients. Some scholars also believe that the hippocampus damage in PTSD patients may be one of the reasons for the persistence of PTSD. Traumatic memories, once formed, are difficult to eliminate, so that people with PTSD often a persistent fear, and fear of eliminating mainly through the prefrontal cortex (mPFC) fear of amygdala response inhibition, assist the mPFC adjust the function of the amygdala, hippocampus, once the hippocampus is damaged, mPFC of amygdala inhibitory effect will be weakened [23].

\section{Influence of Stress Trauma on Dementia}

Clement et al. [24] showed that negative life events may be one of the risk factors for the onset of Alzheimer's disease (AD), and there is a certain correlation between early mental trauma and the onset of AD. Qureshi et al. [25] found that the incidence of dementia in veterans with PTSD was significantly higher than that in non-PTSD veterans. Tsolaki et al. [26] confirmed that over $70 \%$ of $\mathrm{AD}$ patients had experienced at least one severe traumatic event prior to the diagnosis of $\mathrm{AD}$, versus only $55 \%$ of the control subjects. A case-control study by Shen Yuyou et al. [27] showed that $\mathrm{AD}$ was associated with the occurrence of major life events (spouse death, divorce, and child death). In a study of 93 patients with PTSD who survived the Holocaust in Germany, 13 patients developed dementia, of which the incidence of vascular dementia was $66 \%$, the incidence of AD was $23 \%$, and other dementia subtypes were $11 \%$ [28]. PTSD has not been found to be associated with a specific subtype of dementia, being associated with almost all types of dementia, especially vascular dementia and AD. Although the damage of cognitive function in people with PTSD that has been a large number of studies confirm that but at the moment about PTSD and the forward of the correlation between cognitive decline is lack of system research. PTSD patients early cognitive function change whether can be as early signs of dementia or as an independent risk factor for dementia remains to be further research.

\section{The Effect of Stress Trauma on Cognition}

Traumatic events often lead to significant physiological and psychological responses of individuals, increase the risk of mental and psychosomatic diseases [29], and may also cause cognitive decline [30]. Peavy et al. [31] found that the more traumatic events experienced, the higher the risk of cognitive impairment. Traumatic events can have long-lasting effects on the human brain that can be delayed decades or even a lifetime after the stress is experienced. Previous research data have shown that both acute trauma and long-term chronic stress may affect individual learning and memory functions, and most of the results 
are from animal experiments and observational studies [32]. Aggarwal et al. [33] conducted a 7-year follow-up study involving 6200 adults and found that the more stressful events experienced, the greater the impact on cognitive function and the faster the rate of cognitive decline. People who suffer from PTSD after trauma in adulthood are also at increased risk of cognitive impairment [34]. It is concluded that severe traumatic events in adulthood are one of the risk factors for long-term cognitive decline.

\section{Summarize}

1) Major mental trauma is the main cause of memory impairment in PTSD patients, and the more severe the trauma symptoms are, the more severe the memory impairment is.

2) The frontal abstract thinking and memory ability of PTSD patients are severely impaired, and the patients generally have executive dysfunction, which has no correlation with the clinical symptoms of PTSD, suggesting that the executive dysfunction of PTSD patients is not affected by other factors and is one of the independent neurological symptoms.

3) Cognitive decline in PTSD patients may be one of the important reasons for the impairment of memory function, and cognitive decline is also the result of cognitive impairment caused by primary symptoms of PTSD.

4) PTSD patients have significant memory impairment, including visual regeneration, comprehension memory, delayed memory and visual regeneration disorders, and their memory impairment is more serious than that of patients with depression and anxiety.

\section{Conclusion}

Despite the rapid development of science and technology and economy, people are still suffering from a variety of natural and man-made disasters and continuous wars. Focusing on the severe international situation, the research on PTSD has attracted much attention both at home and abroad. Researchers have explored through the neuroendocrine, molecular biology, behavioral and other aspects. The continuous development of imaging technologies, functional imaging technologies, including MRI/fMRI, PET/ PET-CT and EGG, have provided strong support for us to explore the brain functional mechanism of PTSD. The relationship between PTSD and the impairment of long-term cognitive function still needs to be further systematically studied. Addressing mental factors and giving psychological intervention in advance, cognitive dysfunction can be controlled in the earliest stage of disease development, in order to reduce various mental disorders in the long term.

\section{Funding}

The work was supported by 2021 Natural Science Foundation of Hainan Province (No. 820QN395). 


\section{Conflicts of Interest}

The authors declare no conflicts of interest regarding the publication of this paper.

\section{References}

[1] Howell (2008) Psychiatry. 6th Edition, People's Medical Publishing House, Beijing 140.

[2] Yaffe, K., Vittinghoff, E., Lindquist, K., Barnes, D., Covinsky, K.E., Neylan, T., et al. (2010) Posttraumatic Stress Disorder and Risk of Dementia among US Veterans. Archives Of General Psychiatry, 67, 608-613. https://doi.org/10.1001/archgenpsychiatry.2010.61

[3] Qureshi, S.U., Kimbrell, T., Pyne, J.M., Magruder, K.M., Hudson, T.J., Petersen, N.J., et al. (2010) Gremer Prevalence and Incidence of Dementia in Older Veterans with Posttraumatic Stress Disorder. Journal of the American Geriatrics Society, 58, 1627-1633. https://doi.org/10.1111/j.1532-5415.2010.02977.x

[4] Childress, J.E., McDowell, E.J., Dalai, V.V., Bogale, S.R., Ramamurthy, C., Jawaid, A., et al. (2013) Hippocampal Volumes in Patients with Chronic Combat-Related Posttraumatic Stress Disorder: Asystematic Review. The Journal of Neuropsychiatry and Clinical Neurosciences, 25, 12-25.

https://doi.org/10.1176/appi.neuropsych.12010003

[5] Qi, R.F. and Lu, G.M. (2012) Progress of Brain Functional Neuroimaging Research in Patients with Post-Traumatic Stress Disorder. Journal of Medical Imaging, 22, 1023-1025.

[6] Brunetti, M., Sepede, G., Mingoia, G., Catani, C., Ferretti, A., Merla, A., et al. (2010) Elevated Response of Human Amygdala to Neutral Stimuli in Mild Post Traumatic Stress Disorder: Neural Correlates of Generalized Emotional Response. Neuroscience, 168, 670-679. https://doi.org/10.1016/j.neuroscience.2010.04.024

[7] Liu, M.Q., Wu, Q.Z., Li, J., et al. (2012) A Study of Amygdala Magnetic Resonance Imaging in Children with Post-Traumatic Stress Disorder. Radiology Practice, 27, 702-705.

[8] Castillo, M., Kwock, L. and Mukherji, S.K. (1996) Clinical Applications of Proton MR Spectroscopy. American Journal of Neuroradiology, 17, 1-15.

[9] Karl, A., Schaefer, M., Malta, L.S., Dörfel, D., Rohleder, N. and Werner, A. (2006) A Meta-Analysis of Structural Brain Abnormalities in PTSD. Neuroscience \& Biobehavioral Reviews, 30, 1004-1031. https://doi.org/10.1016/j.neubiorev.2006.03.004

[10] Rauch, S.L., Shin, L.M. and Phelps, E.A. (2006) Neurocircuitry Models of Posttraumatic Stress Disorder and Extinction: Human Neuroimaging Research-Past, Present, and Future. Biological Psychiatry, 60, 376-382.

https://doi.org/10.1016/j.biopsych.2006.06.004

[11] Zhang, Y., Zhang, J.N., Qiu, M.G., Xie, B., Chen, H., Li, M., et al. (2012) Research on the Regional Coherence of Post-Traumatic Stress Disorder in the Brain. Journal of the Third Military Medical University, 34, 2260-2262.

[12] Zhu, H.R., Zhang, J.R., Qiu, C.J., Wu, R.Z., Meng, Y.J., Cui, H.F., et al. (2012) A Study on Local Coherence of Brain Function in Patients with Post-Traumatic Stress Disorder. West China Medical Journal, 27, 641-645.

[13] Daniels, J.K., Coupland, N.J., Hegadoren, K.M., Rowe, B.H., Densmore, M., Neufeld, R.W.J., et al. (2012) Neural and Behavioral Correlates of Peritraumatic Dissociation in an Acutely Traumatized Sample. Journal of Clinical Psychiatry, 73, 
420-426. https://doi.org/10.4088//CP.10m06642

[14] Yin, Y., Li, L., Jin, C., Hu, X., Duan, L., Eyler, L.T., et al. (2011) Abnormal Base Line Brain Activity in Posttraumatic Stress Disorder: Aresting-State Functional Magnetic Resonance Imaging Study. Neuroscience Letters, 498, 185-189. https://doi.org/10.1016/j.neulet.2011.02.069

[15] Wu, R.Z., Zhang, J.R., Qiu, C.J., Meng, Y.J., Zhu, H.R., Gong, Q.Y., et al. (2011) Study on Resting-State Default Mode Network in Patients with Posttraumatic Stress Disorder after the Earthquake. Journal of Sichuan University (Medical Science Edition), 42, 397-400.

[16] Gusnard, D.A. and Raichle, M.E. (2001) Searching for a Baseline: Functional Imaging and the Resting Human Brain. Nature Reviews Neuroscience, 2, 685-694. https://doi.org/10.1038/35094500

[17] Halari, R., Simic, M., Pariante, C.M., Papadopoulos, A., Cleare, A., Brammer, M., et al. (2009) Reduced Activation in Lateral Prefrontal Cortex and Anterior Cingulate during Attention and Cognitive Control Functions in Medication-Nave Adolescents with Depression Compared to Controls. Journal of Child Psychology and Psychiatry, 50, 307-316. https://doi.org/10.1111/j.1469-7610.2008.01972.x

[18] Ni, W., Shao, Y.C., Ye, E.M., Tan, S.W. and Yang, Z. (2013) Effects of Chronic Stress on Power Spectrum and Functional Symmetry of EEG Alpha Wave. Military Medicine, 37, 898-902.

[19] Gong, L., Liu, Z.M., Li, B., Chen, Y. and Zhou, J. (2014) Study on Memory Impairment and Brain Structural Magnetic Resonance Characteristics of Post-Traumatic Stress Disorder. Chinese Journal of Practical Neurological Diseases, 17, 93-94.

[20] Mostaid, M.S., Mancuso, S.G., Liu, C., et al. (2017) Meta-Analyses Revealed Associations between Genetic Variation in the 5' and 3' Regions of Neuregulin-1 and Neuregulin-1 Schizophrenia. Translational Psychiatry volume, 7, e1004. https://doi.org/10.1038/tp.2016.279

[21] Guo, M., Guo, J.C., Chen, F., Jiang, X.L. and Li, J.J. (2012) A Study of Hippocampal Volume and Magnetic Resonance Spectrum in Patients with Post-Traumatic Stress Disorder. Chinese Journal of Health Psychology, 20, 1441-1443.

[22] Pruessner, J.C., Collins, D.L., Pruessner, M. and Evans, A.C. (2001) Age and Gender Predict Volume Decline in Theanterior and Posterior Hippocampus in Early Adulthood. Journal of Neuroscience, 21, 194-200. https://doi.org/10.1523/JNEUROSCI.21-01-00194.2001

[23] He, J.W., Shang, Z.L., Liu, W.Z. and Wang, W. (2014) Brain Mechanisms Related to Fear Cancellation. Chinese Journal of Health Psychology, 22, 1593-1595.

[24] C1ement, J.P., Darthout, N., Nubukpo, P. (2003) Life Events, Personality and Dementia. Psychologie \& Neuropsychiatrie du Vieillissement, 1, 129-138

[25] Qureshi, S.U., Long, M.E., Bradshaw, M.R., Pyne, J.M., Magruder, K.M., Kimbrell, T., et al. (2011) Does PTSD Impair Cognition beyond the Effect of Trauma. The Journal of Neuropsychiatry and Clinical Neurosciences, 23, 16-28. https://doi.org/10.1176/appi.neuropsych.23.1.16

[26] Tsolaki, M., Papaliagkas, V., Kounti, F., Messini, C., Boziki, M., Anogianakis, G., et al. (2010) Severely Stressful Events and Dementia: A Study of an Elderly Greek Demented Population. Psychiatry Research, 176, 51-54.

https://doi.org/10.1016/j.psychres.2009.06.001

[27] Shen, Y.B., Li, Y.T., Li, G., et al. (1992) A Case-Control Study on Risk Factors of Alzheimer's Disease: Multicenter Collaborative Study in China. Chinese Journal of 
Neuropsychiatry, 25, 284-287.

[28] Sperling, W.j., Kreil, S.K. and Biermann, T. (2011) Posttraumatic Stress Disorder and Dementia in Holocaust Survivors. The Journal of Nervous and Mental Disease, 199, 196-198. https://doi.org/10.1097/NMD.0b013e31820c71e0

[29] Pesonen, A.K., Eriksson, J.G., Heinonen, K., Kajantie, E., Tuovinen, S., Alastalo, H., et al. (2013) Cognitive Ability and Decline after Early Life Stress Exposure. Neurobiology of Aging, 34, 1674-1679.

https://doi.org/10.1016/j.neurobiolaging.2012.12.012

[30] Thirunavukkarasu, P., Vijayakumari, A., John, J.P., et al. (2014) Dysbindin and Neuregulin Gene Polymorphisms on Brain Morphometry in Schizophrenia and Schizophrenia in Healthy Subjects from South India. Asian Journal of Psychiatry, 10, 62-68. https://doi.org/10.1016/j.ajp.2014.04.002

[31] Alastalo, H., von Bonsdorff, M.B., Räikkönen, K., Pesonen, A.-K., Osmond, C., Barker, D.J.P., et al. (2013) Early Life Stress and Physical and Psychosocial Functioning in Late Adulthood. PLoS ONE, 8, e69011.

https://doi.org/10.1371/journal.pone.0069011

[32] Peavy, G.M., Jacobson, M.W., Salmon, D.P., Gamst, A.C., Patterson, T.L., Goldman, S., et al. (2012) The Influence of Chronic Stress on Dementia-Related Diagnostic Change in Older Adults. Alzheimer Disease \& Associated Disorders, 26, 260-266. https://doi.org/10.1097/WAD.0b013e3182389a9c

[33] Aggarwal, N.T., Wilson, R.S., Beck, T.L., Rajan, K.B., Mendes de Leon, Carlos, F., Evans, D.A., et al. (2014) Perceived Stress and Change in Cognitive Function among Adults 65 Years and Older. Psychosomatic Medicine, 76, 80-85. https://doi.org/10.1097/PSY.0000000000000016

[34] Schwabe, L., Joels, M., Roozendaal, B., Wolf, O.T., Oitzl, M.S., et al. (2012) Stress Effects on Memory: An Update and Integration. Neuroscience \& Biobehavioral Reviews, 36, 1740-1749. https://doi.org/10.1016/j.neubiorev.2011.07.002 